\title{
Axial anomaly generation by domain wall motion in Weyl semimetals
}

\author{
Julia D. Hannukainen $\odot,{ }^{1}$ Yago Ferreiros $\odot,{ }^{1,2}$ Alberto Cortijo,${ }^{3}$ and Jens H. Bardarson ${ }^{1}$ \\ ${ }^{1}$ Department of Physics, KTH Royal Institute of Technology, 10691 Stockholm, Sweden \\ ${ }^{2}$ IMDEA Nanociencia, Faraday 9, 28049 Madrid, Spain \\ ${ }^{3}$ Instituto de Ciencia de Materiales de Madrid, CSIC, Cantoblanco, 28049 Madrid, Spain
}

(Received 27 December 2019; accepted 16 November 2020; published 1 December 2020)

\begin{abstract}
A space-time dependent node separation in Weyl semimetals acts as an axial vector field. Coupled with domain wall motion in magnetic Weyl semimetals, this induces axial electric and magnetic fields localized at the domain wall. We show how these fields can activate the axial (chiral) anomaly and provide a direct experimental signature of it. Specifically, a domain wall provides a spatially dependent Weyl node separation and an axial magnetic field $\boldsymbol{B}_{5}$, and domain wall movement, driven by an external magnetic field, gives the Weyl node separation a time dependence, inducing an axial electric field $\boldsymbol{E}_{5}$. At magnetic fields beyond the Walker breakdown, $\boldsymbol{E}_{5} \cdot \boldsymbol{B}_{5}$ becomes nonzero and activates the axial anomaly that induces a finite axial charge density-imbalance in the number of left- and right-handed fermions-moving with the domain wall. This axial density in turn produces, via the chiral magnetic effect, an oscillating current flowing along the domain wall plane, resulting in a characteristic radiation of electromagnetic waves emanating from the domain wall. A detection of this radiation would constitute a direct measurement of the axial anomaly induced by axial electromagnetic fields.
\end{abstract}

DOI: 10.1103/PhysRevB.102.241401

Introduction. The smallest number of Weyl fermions realizable as quasiparticles in a crystal is two [1,2]—one left handed and one right handed. In the presence of inversion symmetry, we can choose the origin of momentum space such that one Weyl fermion resides at $\boldsymbol{b}$ and the other at $\boldsymbol{- b}$. Since time reversal does not change the handedness of a Weyl fermion, such a minimal Weyl semimetal necessarily breaks time-reversal symmetry $[3,4]$. The Weyl node splitting $2 \boldsymbol{b}$ is then induced by the time-reversal breaking and can be thought of as a magnetization. Such a magnetic Weyl semimetal was recently realized in $\mathrm{EuCd}_{2} \mathrm{As}_{2}$ at intermediate temperatures $[5,6]$ and in $\mathrm{EuCd}_{2} \mathrm{Sb}_{2}$ in an external magnetic field [7]; several further Weyl states in magnetic materials were experimentally observed [8-11].

The electronic response of the Weyl fermions to external electromagnetic fields is fundamentally influenced by the chiral anomaly $[12,13]$. The handedness of the Weyl fermions is not generally conserved and the axial density $n_{5}=n_{L}-$ $n_{R}$, the difference in density of left- and right-handed Weyl fermions, instead satisfies the homogeneous anomaly equation [14]

$$
\partial_{t} n_{5}=\frac{e^{2}}{2 \hbar^{2} \pi^{2}}\left(\boldsymbol{E} \cdot \boldsymbol{B}+\frac{1}{3} \boldsymbol{E}_{5} \cdot \boldsymbol{B}_{5}\right) .
$$

Published by the American Physical Society under the terms of the Creative Commons Attribution 4.0 International license. Further distribution of this work must maintain attribution to the author(s) and the published article's title, journal citation, and DOI. Funded by Bibsam.
Here, $\boldsymbol{E}$ and $\boldsymbol{B}$ are the usual electric and magnetic fields, while $\boldsymbol{E}_{5}$ and $\boldsymbol{B}_{5}$ are so-called axial electric and magnetic fields $[15,16]$, which point in the opposite direction for the two chiralities. Direct experimental signatures of the anomaly have proven hard to come by. While negative magnetoresistance is a consequence of the chiral anomaly $[17,18]$, it is not an unambiguous signature of it [19-23]. Axial fields are also challenging to realize as they may require systematic and significant straining of materials [24-26]; obtaining an axial electric field $\boldsymbol{E}_{5}$ is particularly hard, as this requires controllable time-dependent strain. This is because the Weyl node separation $\boldsymbol{b}$ couples to the Weyl fermions as an axial vector potential and strain gives it a space-time dependence as $\boldsymbol{b} \rightarrow \boldsymbol{b}(\boldsymbol{r}, t)$. This then gives rise to axial fields through $\boldsymbol{B}_{5}=$ $\boldsymbol{\nabla} \times \boldsymbol{b}$ and $\boldsymbol{E}_{5}=-\partial_{t} \boldsymbol{b}$, in analogy with how electromagnetic fields are obtained from a vector potential [27].

In this Rapid Communication we discuss how both of these difficulties - the generation of axial fields and detection of the axial anomaly - are overcome by studying domain wall motion [28,29] in Weyl semimetals. Indeed, in a magnetic Weyl semimetal a space-time variation in the Weyl node separation is naturally realized at domain walls in the magnetization [30]. Such domain walls have been indirectly observed, for example, in the magnetic nodal semimetal CeAlGe [8]. Domain wall motion has also been studied in related systems such as junctions of ferromagnets and topological insulators [31-35], and the interplay of anomalies and skyrmion motion in $\mathrm{He}_{3}$ has been experimentally observed [36].

Consider a magnetic domain wall along the $x$ direction, pointing in the $\pm z$ direction deep in the bulk, as depicted in Fig. 1. For concreteness, we assume the easy axis of the magnetic anisotropy to be in the $z$ direction and the hard-axis anisotropy to lie in the $y$ direction, making the $x z$ plane the 


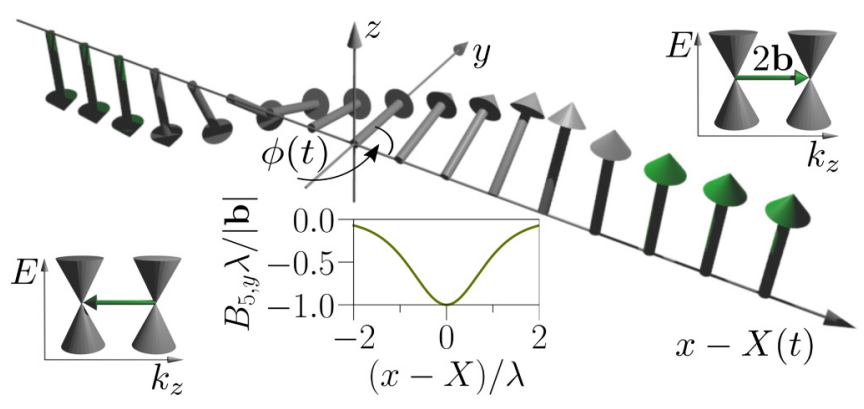

FIG. 1. A domain wall along the $x$ direction with a continuously varying Weyl node separation $2 \boldsymbol{b}$. The two insets with Weyl cones show the corresponding Weyl node separation in momentum space, given by the bulk magnetization vector (green). The domain wall has a hard-axis anisotropy in the $y$ direction, and an easy axis in the $z$ direction. $\phi(t)$ is the angle of the magnetization at the domain wall center out of the easy-axis plane ( $x z$ plane), and $X(t)$ is the position of the domain wall center. The domain wall depicted is in the Bloch configuration for which $\phi(t)=\pi / 2$. The Bloch wall only has a nonzero component of $\boldsymbol{B}_{5}$ in the $y$ direction, and $B_{5, y} \lambda /|\boldsymbol{b}|$ is plotted as a function of $x-X(0)$ (middle bottom inset); for parameter values, see Ref. [37].

easy plane. The domain wall can be described in terms of two collective coordinates, the position $X(t)$ of the center of the wall and the internal angle $\phi(t)$, which measures the angle of the magnetization out of the easy plane. $X(t)$ and $\phi(t)$ describe zero modes of fluctuations around the domain wall arising from translation invariance along the $x$ direction and rotation invariance around the $z$ axis, respectively [38]. While the existence of a hard-axis anisotropy formally breaks the rotational invariance, $X(t)$ and $\phi(t)$ are still good collective coordinates in the limit of weak anisotropy. There are two special configurations of the domain wall, the Néel wall for which $\phi=0$ where the domain wall is situated in the easy plane, and the Bloch wall, for which $\phi=\pi / 2$, illustrated in Fig. 1. Since $\boldsymbol{b}$ rotates from $-b \hat{z}$ to $b \hat{z}$ an axial magnetic field localized at the domain wall is obtained. This is similar to the $\boldsymbol{B}_{5}$ obtained at the surface of Weyl semimetals [39], except that it is not constrained to a definite location in space.

When the domain wall moves, the magnetization becomes time dependent, generating an axial electric field $\boldsymbol{E}_{5}$. A controllable way of moving a domain wall is by a magnetic field $\boldsymbol{B}=B \hat{z}$. This results in a rigid shift of the domain wall center $X(t)$ with an average velocity that increases linearly with $B$ up until a critical value $B_{c}$, at which the internal angle starts rotating and the velocity decreases-this is called the Walker breakdown [40]. The axial electric field generated in this movement is a function of both the rotation and the velocity of the domain wall. However, as we show, the axial anomaly (which depends on $\boldsymbol{E}_{5} \cdot \boldsymbol{B}_{5}$ ) is only activated when the internal angle starts rotating, for magnetic fields larger than $B_{\mathrm{c}}$. Once it is activated, an axial density $n_{5}$, localized at the domain wall, builds up and an oscillating current is induced, via the chiral magnetic effect [41]. This results in electromagnetic radiation which is a direct signature of the axial anomaly induced by axial fields.

Domain wall dynamics. We take the Weyl node separation in a domain wall to define a unit magnetization $\boldsymbol{m}$ as $\boldsymbol{b}(\boldsymbol{r}, t)=$
$\Delta /\left(e v_{F}\right) \boldsymbol{m}(\boldsymbol{r}, t)$, where $e$ is the elementary charge, $v_{F}$ the Fermi velocity, and $\Delta$ an effective exchange coupling between the electrons and the magnetization. The variation of $\boldsymbol{b}$ with $\boldsymbol{r}$ and $t$ is slow enough, compared to typical electronic time and length scales, that the interpretation of it as a Weyl node separation in momentum space still makes sense. Expressed in the collective coordinates,

$\boldsymbol{m}=\left[\frac{\cos (\phi(t))}{\cosh \left(\frac{x-X(t)}{\lambda}\right)}, \frac{\sin (\phi(t))}{\cosh \left(\frac{x-X(t)}{\lambda}\right)},-q \tanh \left(\frac{x-X(t)}{\lambda}\right)\right]$,

where $\lambda$ is the domain wall width and $q= \pm 1$ is the topological charge [38]; we consider the case $q=-1$ (cf. Fig. 1). The dynamics of the domain wall is encapsulated in a ferromagnetic action $S_{\mathrm{FM}}=\int d t\left(L_{\mathrm{B}}-H_{\mathrm{H}}-\right.$ $H_{\mathrm{Z}}$ ) which considers the precession and exchange coupling of the magnetization, coupled to an external magnetic field [28]. The Lagrangian describing the precession is given by a Berry phase term $L_{\mathrm{B}}=\hbar / a^{3} \int d^{3} x \dot{\phi}(\cos \theta-$ $1)$, where $\theta=2 \tan ^{-1} \exp [-(x-X(t)) / \lambda]$ and $a$ is the lattice constant [42]. The exchange coupling contributes the term $H_{\mathrm{H}}=1 /\left(2 a^{3}\right) \int d^{3} x\left(J a^{2}|\nabla \boldsymbol{m}|^{2}-K m_{z}^{2}+K_{\perp} m_{y}^{2}\right)$, which is the Heisenberg Hamiltonian in the continuous limit. Here, $J, K$, and $K_{\perp}$ are positive constants: $J$ is the exchange energy, and $K$ and $K_{\perp}$ are the easy- and hard-axis anisotropy energies. The contribution from an external magnetic field $\boldsymbol{B}=B \hat{z}$, applied in the direction of the easy-axis anisotropy, is included as a Zeeman term, $H_{Z}=\hbar / a^{3} \int d^{3} x \boldsymbol{m} \cdot \gamma \boldsymbol{B}$, where $\gamma$ is the electron gyromagnetic ratio.

The collective coordinate description of the domain wall in terms of $X(t)$ and $\phi(t)$ is valid as long as there is translational invariance in the $x$ direction and rotational invariance around the $z$ direction. While the existence of a hard-axis anisotropy would deform the domain wall and break the rotational invariance, the deformation is negligible in the limit $K_{\perp} \ll K$, in which $X(t)$ and $\phi(t)$ are good collective coordinates [29]. While this is not an essential limit, it simplifies our discussion so we assume it henceforth. The domain wall action in this limit in terms of collective coordinates [28],

$$
S_{\mathrm{FM}}=-\frac{2 \hbar A}{a^{3}} \int d t\left(\dot{\phi} X+v_{\perp} \sin ^{2} \phi-\gamma B X\right) .
$$

Here, $A$ is the cross section of the sample in the $y z$ plane and $\nu_{\perp}=\lambda K_{\perp} /(2 \hbar)$, with $\lambda=\sqrt{J / K}$ the domain wall width. The first term is the Berry phase term, the second the contribution from the Heisenberg Hamiltonian, and the last the Zeeman term. The time evolution of the collective coordinates is given by the action $S_{\mathrm{FM}}$ together with damping, which takes into account magnetization relaxation effects. Incorporating the damping as a dissipation function $W=-\hbar A \lambda \alpha / a^{3}\left[(\dot{X} / \lambda)^{2}+\right.$ $\dot{\phi}^{2}$ ], where $\alpha$ is the Gilbert damping constant [43], the generalized Euler-Lagrange equations of motion take the form

$$
\begin{gathered}
\dot{\phi}+\frac{\alpha}{\lambda} \dot{X}=\gamma B, \\
\dot{X}-\alpha \lambda \dot{\phi}=v_{\perp} \sin 2 \phi,
\end{gathered}
$$

which are combined into a single equation for the internal angle: $\dot{\phi}=a_{1}-a_{2} \sin (2 \phi)$. Here, $a_{1}=\gamma B /\left(\alpha^{2}+1\right)$ and $a_{2}=$ $\alpha v_{\perp} /\left[\left(\alpha^{2}+1\right) \lambda\right] . a_{2}$ is always positive while the sign of $a_{1}$ depends on the direction of the magnetic field. 
The solutions for $\phi(t)$ depend on the magnitude of $B$ and are divided into two domains separated by the critical magnetic field $B_{\mathrm{c}}=\alpha v_{\perp} /(\gamma \lambda)$ obtained when $\left|a_{1}\right|=a_{2}$. This is observed from the solution for $\phi(t)$, which for an initial condition $\phi(0)=0$ is

$$
\tan (\phi)=\frac{a_{1} \tan \left(\sqrt{a_{1}^{2}-a_{2}^{2}} t\right)}{\sqrt{a_{1}^{2}-a_{2}^{2}}+a_{2} \tan \left(\sqrt{a_{1}^{2}-a_{2}^{2}} t\right)} .
$$

The square root in the above expression is negative when $B<$ $B_{\mathrm{c}}$, in which case the solution is $\tan (\phi)=a_{1} \tanh (\zeta t) /[\zeta+$ $\left.a_{2} \tanh (\zeta t)\right]$, where $\zeta=\sqrt{a_{2}^{2}-a_{1}^{2}}$. In the long-time limit $t \rightarrow$ $\infty$, this results in a constant angle $\phi=1 / 2 \arcsin \left(B / B_{\mathrm{c}}\right)$. The domain wall velocity is also constant in this limit: $\dot{X}=$ $\lambda \gamma B / \alpha$. When the magnetic field is larger than the critical value, $B>B_{\mathrm{c}}$, the internal angle oscillates in time according to $\phi(t)=\arctan \left\{a_{1} \tan (\omega t) /\left[\omega+a_{2} \tan (\omega t)\right]\right\}$, with the angular frequency $\omega=\sqrt{a_{1}^{2}-a_{2}^{2}}$. In this regime the domain wall position $X(t)=[-\phi(t)+\gamma B t] \lambda / \alpha$ also increases with time with an oscillatory motion. The magnitude of the magnetic field therefore plays a role in how the domain wall moves, which has implications for the onset of the chiral anomaly. The anomaly equation (1) (with $\boldsymbol{E}=\mathbf{0}$ ) is proportional to

$$
\boldsymbol{E}_{5} \cdot \boldsymbol{B}_{5}=\frac{\Delta^{2}}{e^{2} v_{F}^{2} \lambda} \frac{\dot{\phi} \cos \phi}{\cosh ^{3}\left(\frac{x-X(t)}{\lambda}\right)},
$$

which is zero when $B<B_{\mathrm{c}}$, implying that the chiral anomaly is only activated in the Walker breakdown regime $B>B_{\mathrm{c}}$. The axial electric field, which contributes with the term $\dot{\phi}$, is also nonzero before the Walker breakdown, but is then orthogonal to $\boldsymbol{B}_{5}$.

The axial chemical potential. The axial anomaly induced by the domain wall motion generates an axial chemical potential $\mu_{5}=\left(\mu_{L}-\mu_{R}\right) / 2$, with $\mu_{L}$ and $\mu_{R}$ the chemical potentials of left- and right-handed Weyl fermions, respectively. The anomaly equation is of the form $\partial_{t} n_{5}=e^{2} /\left(6 \hbar^{2} \pi^{2}\right) \boldsymbol{E}_{5} \cdot \boldsymbol{B}_{5}-$ $n_{5} / \tau$, where the second term takes into account intervalley scattering between the two Weyl cones, with intervalley scattering time $\tau[44,45]$ and where $\boldsymbol{E}_{5} \cdot \boldsymbol{B}_{5}$ oscillates in time with period $\tau_{\phi}=2 \pi / \omega$. In the limit $\tau_{\phi} \ll \tau$, the domain wall oscillates faster than the intervalley scattering and the number density becomes $n_{5}=e^{2} /\left(6 \hbar^{2} \pi^{2}\right) \int_{0}^{t} d s \boldsymbol{E}_{5}(x, s) \cdot \boldsymbol{B}_{5}(x, s)$.

The axial chemical potential is considered to be space and time dependent and relates to the axial number density as

$$
\mu_{5}(x, t)=\frac{2^{\frac{2}{3}}\left(\left[C_{1} n_{5}+\sqrt{C_{1}^{2} n_{5}^{2}+4 C_{2}^{3}}\right]^{\frac{2}{3}}-2^{\frac{2}{3}} C_{2}\right)}{6\left(C_{1} n_{5}+\sqrt{C_{1}^{2} n_{5}^{2}+4 C_{2}^{3}}\right)^{\frac{1}{3}}},
$$

where the constants $C_{1}=81 \pi^{2} \hbar^{3} v_{F}^{3}$ and $C_{2}=3\left(3 \mu^{2}+\right.$ $\left.\pi^{2} T^{2} k_{B}^{2}\right), T$ is the temperature, and $k_{B}$ the Boltzmann constant. The above expression for $\mu_{5}$ holds in the limit of small magnetic fields, $\hbar e B \ll \mu_{5}^{2} / \nu_{F}^{2}$, where $\mu=\left(\mu_{L}+\mu_{R}\right) / 2$ is the average chemical potential [46]. $\mu_{5}(x, t)$ oscillates in time, is located at the domain wall, and travels along the $x$ direction as $X(t)$ evolves with time (see Fig. 2).

Measuring the anomaly. The axial chemical potential generates a current density [47] proportional to the external
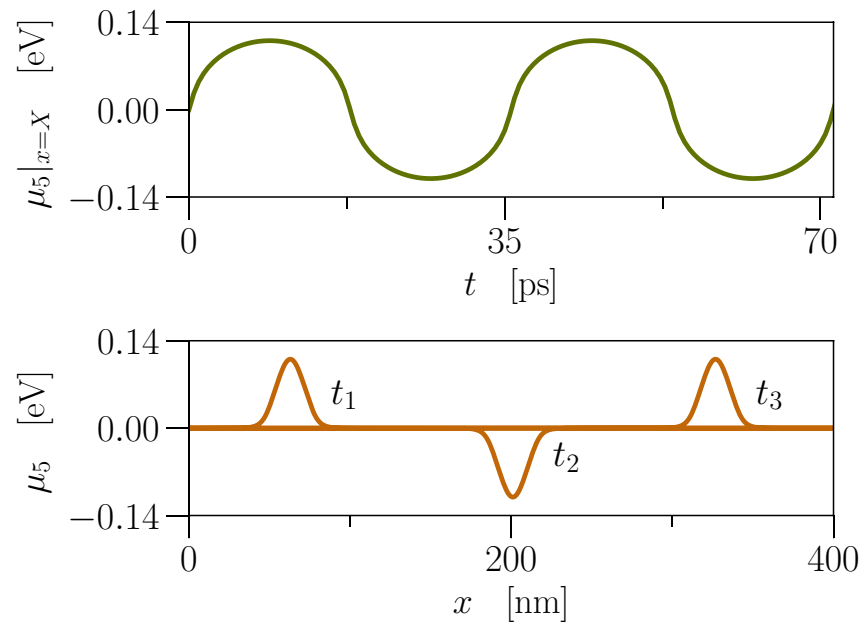

FIG. 2. Axial chemical potential $\mu_{5}$ as function of time $t$, evaluated at the domain wall center $x=X(t)$ (upper panel) and as a function of $x$ at times $t_{1}=100 \tau_{\phi}+\tau_{\phi} / 4, t_{2}=320 \tau_{\phi}-\tau_{\phi} / 4$, and $t_{3}=520 \tau_{\phi}+\tau_{\phi} / 4$ (lower panel). Here, we take the chemical potential $\mu=10 \mathrm{meV}$, temperature $T=300 \mathrm{~K}$, and magnetic field $B=1$ $\mathrm{T}$; other parameters are given in Ref. [37].

magnetic field through the chiral magnetic effect [46],

$$
J^{A}(x, t)=\frac{e^{2}}{2 \pi^{2} \hbar^{2}} \mu_{5}(x, t) B \hat{z} \equiv J_{z}^{A}(x, t) \hat{z}
$$

The rotation of the magnetization further yields an effective current, $\boldsymbol{J}^{M}(x, t)=\boldsymbol{\nabla} \times \boldsymbol{M}$, where $\boldsymbol{M}=\gamma \hbar / a^{3} \boldsymbol{m}$ is the magnetization density. The currents $J_{z}^{A}(x, t)$ and $\boldsymbol{J}^{M}(x, t)$ give rise to electromagnetic fields, measurable through their radiated power. We describe these fields using Jefimenko's equations [48], and consider them separately in the near-field, $r \ll R_{0}$, and the far-field, $r \gg R_{0}$, limits, where $R_{0}$ is the wavelength of the electromagnetic fields and $r=\sqrt{[x-X(t)]^{2}+y^{2}+z^{2}}$ is the distance to the detector from the domain wall center [49]. In both limits the domain size is considered to be the smallest of the three length scales, $\lambda \ll r, R_{0}$. We further require that $L_{z}>v_{F} \tau_{\phi}$, where $L_{z}$ is the width of the sample in the $z$ direction (in the opposite limit accumulation of charge at the edge of the sample might become relevant), which for realistic parameters [37] and $B \gtrsim 1 \mathrm{~T}$ holds when $L_{z} \sim 10 \mu \mathrm{m}$. The radiation in the near field is measurable by currenttechnology on-chip, which can detect weak signals of only a few emitted photons [50]. Reactive components dominate the electromagnetic fields in the near field, and by describing the electromagnetic fields in this limit as an expansion in $r / c$, we find that the only radiative contribution up to second order in $r / c$ originates from the anomaly current. The resulting power impinging on a detector of size $\ell \times \ell$, and a small solid angle, is given by $P_{\ell}^{A}(\theta, \varphi)=\ell^{2} d_{2}^{2} B^{2} \lambda^{2}\left\langle\left(\partial_{t}^{2} \mu_{5}\right)^{2}\right\rangle \sin ^{2} \theta /\left(2 \varepsilon_{0} c^{5}\right)$, where $d_{2}=L_{y} L_{z} e^{2} /\left(8 \pi^{3} \hbar^{2}\right), \varepsilon_{0}$ is the vacuum permeability, $\theta, \varphi$ are the polar and azimuthal angles, and $\langle\cdots\rangle$ refers to the time average [51]. Figure 3 depicts the number of photons, $n=P_{\ell}^{A} t_{\ell} / E_{p}$, emitted by a domain wall during the time $t_{\ell}=\ell /\langle\dot{X}\rangle$ it takes it to traverse the detector length $\ell=1 \mu \mathrm{m}$, as a function of the external magnetic field, where $E_{p}=\hbar \omega$ is the photon energy. The number of emitted photons goes above 1 for $B \sim 2.5 \mathrm{~T}$ and increases as $B^{4}$, yielding around 1530 


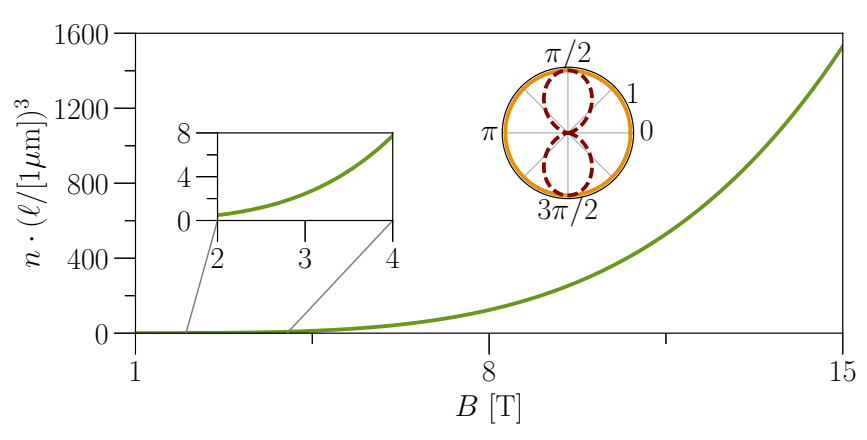

FIG. 3. The number of photons $n=P_{\ell, \max }^{A} t_{\ell} / E_{p}$ emitted by the domain wall during the time $t_{\ell}=\ell /\langle\dot{X}\rangle$ it traverses the detector length $\ell=1 \mu \mathrm{m}$, as a function of external magnetic field $B$. Here, $P_{\ell, \max }^{A}=\ell^{2} d_{2}^{2} B^{2} \lambda^{2}\left\langle\left(\partial_{t}^{2} \mu_{5}\right)^{2}\right\rangle \sin ^{2} \theta /\left.\left(2 \varepsilon_{0} c^{5}\right)\right|_{\theta=\pi / 2}$ is the radiated power in the near field, and is due to the anomaly current, and $E_{p}=\hbar \omega$ is the photon energy. The polar plot depicts the normalized power per solid angle, $\left(d P^{A} / d \Omega\right) / \mathcal{A}=\sin ^{2} \theta$, where $\mathcal{A}$ is the amplitude of $\left(d P^{A} / d \Omega\right)$, as a function of the polar angle $\theta$ (maroon, dotted) for any value of the azimuthal angle $\varphi$, and as a function of $\varphi$ (orange, solid) for $\theta=\pi / 2$. Here, $L_{y}=L_{z}=10 \mu \mathrm{m}$, and for other parameter values, see Fig. 2 and Ref. [37].

emitted photons for $B=15 \mathrm{~T}$, making the signal observable by detector sizes $\ell \sim 0.1-1 \mu \mathrm{m}[50,52-54]$.

In the far-field limit the electromagnetic fields radiate, and the power contains contributions from both $J_{z}^{A}(x, t)$ and $J^{M}(x, t)$. The anomaly current is even across the domain wall, while the magnetization current is odd, which results in the radiation due to the magnetization decaying faster with distance than the radiation due to the anomaly (total power due to the anomaly is independent of $r$ while the magnetization power decays as $r^{-2}$ ). For $B \sim 10 \mathrm{~T}$ and $r \sim 1 \mathrm{~cm}$ the contribution to the power due to the anomaly is $10^{4}$ times larger than the magnetization contribution (for details, see Ref. [51]). The power in both limits is thus dominated by the anomaly contribution, and the radiation frequencies range between $\omega / 2 \pi=27$ and $420 \mathrm{GHz}$ for magnetic fields in the interval $B=1-15 \mathrm{~T}$.

These results generalize to multiple domain walls. Adjacent domain walls have opposite topological charge $q= \pm 1$, and therefore travel in opposite directions under the influence of a magnetic field. The current due to the anomaly is independent of the topological charge as the axial chemical potential goes as $q^{2}$. This implies that the radiated field from the anomaly current of two adjacent domain walls add up. The magnetization current, on the other hand, has a $q$ dependence which produces a sign different in the $y$ components of the magnetization current at two adjacent domain walls, which modifies the radiation when considering several domain walls compared to that of a single domain wall. In any case, this will not change the fact that the anomaly contribution is the dominating one, and measurable also in the case of multiple domain walls. However, the radiated power only exists during a finite length of time depending on the velocity of the domain walls, until the domain walls annihilate each other or until they reach the boundary of the sample. Pinning [29] - local enhancement of easy-axis anisotropy, due to, for example, impurities, confining the domain wall to a certain region-could modify the details of the radiation field, since adjacent domain walls could be prevented from annihilating one another and the electromagnetic radiation would come from a fixed location.

Discussion. We have shown how field-driven motion of a domain wall in a magnetic Weyl semimetal leads to the activation of the axial anomaly. This results from the space and time dependent Weyl node separation emerging from the domain wall motion, which generates axial electromagnetic fields. The anomaly generates an axial chemical potential at the domain wall, which in turn results in an oscillating current and electromagnetic microwave radiation, the detection of which would constitute a direct measurement of the axial anomaly. Experimental techniques to detect such microwave radiation are advanced and can even be done on-chip [50,5254]. While we have made some simplifying approximations to highlight the fundamental physics, we expect the qualitative picture to be robust in realistic situations, and a general feature of any domain wall motion in Weyl semimetals. For example, current-driven domain wall motion will lead to the same axial anomaly-triggering mechanism as the one described here, but will allow for an electronic control of anomaly activation, which may be useful in designing experiments and applications. We have also worked in the limit of weak hard-axis anisotropy where a description of the domain wall in terms of collective coordinates is sufficient. Deviations away from this limit will lead to a more complicated theory that needs to take into account modes beyond just the zero modes we include, but this is not expected to modify the qualitative description of the emergence of axial fields located at the domain wall.

We have focused our discussion on the use of domain wall motion for detecting anomaly physics. The other way around, namely the effects of the anomaly on the physics of domain walls and related spintronics phenomena, is an interesting avenue for future studies.

Acknowledgments. This work was supported by the ERC Starting Grant No. 679722. Y.F. acknowledges financial support through the Programa de Atracción de Talento de la Comunidad de Madrid, Grant No. 2018T2/IND-11088. A.C. acknowledges financial support through MINECO/AEI/FEDER, UE Grant No. FIS2015-73454-JIN and European Union structural funds, the Comunidad Autonoma de Madrid (CAM) NMAT2D-CM Program (S2018NMT-4511), and the Ramon y Cajal program through the grant RYC2018-023938-I.
[1] H. B. Nielsen and M. Ninomiya, Absence of neutrinos on a lattice: (I). Proof by homotopy theory, Nucl. Phys. B 185, 20 (1981).
[2] H. B. Nielsen and M. Ninomiya, Absence of neutrinos on a lattice: (II). Intuitive topological proof, Nucl. Phys. B 193, 173 (1981). 
[3] X. Wan, A. M. Turner, A. Vishwanath, and S. Y. Savrasov, Topological semimetal and Fermi-arc surface states in the electronic structure of pyrochlore iridates, Phys. Rev. B 83, 205101 (2011).

[4] N. P. Armitage, E. J. Mele, and A. Vishwanath, Weyl and Dirac semimetals in three-dimensional solids, Rev. Mod. Phys. 90, 015001 (2018).

[5] J.-Z. Ma, S. M. Nie, C. J. Yi, J. Jandke, T. Shang, M. Y. Yao, M. Naamneh, L. Q. Yan, Y. Sun, A. Chikina, V. N. Strocov, M. Medarde, M. Song, Y.-M. Xiong, G. Xu, W. Wulfhekel, J. Mesot, M. Reticcioli, C. Franchini, C. Mudry et al., Spin fluctuation induced Weyl semimetal state in the paramagnetic phase of $\mathrm{EuCd}_{2} \mathrm{As}_{2}$, Sci. Adv. 5, eaaw4718 (2019).

[6] J. R. Soh, F. de Juan, M. G. Vergniory, N. B. M. Schröter, M. C. Rahn, D. Y. Yan, J. Jiang, M. Bristow, P. A. Reiss, J. N. Blandy, Y. F. Guo, Y. G. Shi, T. K. Kim, A. McCollam, S. H. Simon, Y. Chen, A. I. Coldea, and A. T. Boothroyd, Ideal Weyl semimetal induced by magnetic exchange, Phys. Rev. B 100, 201102(R) (2019).

[7] H. Su, B. Gong, W. Shi, H. Yang, H. Wang, W. Xia, Z. Yu, P.-J. Guo, J. Wang, L. Ding, L. Xu, X. Li, X. Wang, Z. Zou, N. Yu, Z. Zhu, Y. Chen, Z. Liu, K. Liu, G. Li, and Y. Guo, Magnetic exchange induced Weyl state in a semimetal $\mathrm{EuCd}_{2} \mathrm{Sb}_{2}$, APL Mater. 8, 011109 (2020).

[8] T. Suzuki, L. Savary, J. P. Liu, J. W. Lynn, L. Balents, and J. G. Checkelsky, Singular angular magnetoresistance in a magnetic nodal semimetal, Science 365, 377 (2019).

[9] I. Belopolski, K. Manna, D. S. Sanchez, G. Chang, B. Ernst, J. Yin, S. S. Zhang, T. Cochran, N. Shumiya, H. Zheng, B. Singh, G. Bian, D. Multer, M. Litskevich, X. Zhou, S.-M. Huang, B. Wang, T.-R. Chang, S.-Y. Xu, A. Bansil et al., Discovery of topological Weyl fermion lines and drumhead surface states in a room temperature magnet, Science $\mathbf{3 6 5}, 1278$ (2019).

[10] D. F. Liu, A. J. Liang, E. K. Liu, Q. N. Xu, Y. W. Li, C. Chen, D. Pei, W. J. Shi, S. K. Mo, P. Dudin, T. Kim, C. Cacho, G. Li, Y. Sun, L. X. Yang, Z. K. Liu, S. S. P. Parkin, C. Felser, and Y. L. Chen, Magnetic Weyl semimetal phase in a Kagomé crystal, Science 365, 1282 (2019).

[11] N. Morali, R. Batabyal, P. K. Nag, E. Liu, Q. Xu, Y. Sun, B. Yan, C. Felser, N. Avraham, and H. Beidenkopf, Fermi-arc diversity on surface terminations of the magnetic Weyl semimetal $\mathrm{Co}_{3} \mathrm{Sn}_{2} \mathrm{~S}_{2}$, Science 365, 1286 (2019).

[12] S. L. Adler, Axial-vector vertex in spinor electrodynamics, Phys. Rev. 177, 2426 (1969).

[13] J. S. Bell and R. Jackiw, A PCAC puzzle: $\pi 0 \rightarrow \gamma \gamma$ in the $\sigma$ model, Nuovo Cimento A 60, 47 (1969).

[14] Throughout this work, this consistent version of the anomaly is the relevant one (cf. Refs. [55,56]).

[15] M. A. H. Vozmediano, M. I. Katsnelson, and F. Guinea, Gauge fields in graphene, Phys. Rep. 496, 109 (2010).

[16] R. Ilan, A. G. Grushin, and D. I. Pikulin, Pseudoelectromagnetic fields in 3D topological semimetals, Nat. Rev. Phys. 2, 29 (2020).

[17] H. B. Nielsen and M. Ninomiya, The Adler-Bell-Jackiw anomaly and Weyl fermions in a crystal, Phys. Lett. B 130, 389 (1983).

[18] D. T. Son and B. Z. Spivak, Chiral anomaly and classical negative magnetoresistance of Weyl metals, Phys. Rev. B 88, 104412 (2013).
[19] J. Xiong, S. K. Kushwaha, T. Liang, J. W. Krizan, M. Hirschberger, W. Wang, R. J. Cava, and N. P. Ong, Evidence for the chiral anomaly in the Dirac semimetal $\mathrm{Na}_{3} \mathrm{Bi}$, Science 350, 413 (2015).

[20] P. Goswami, J. H. Pixley, and S. Das Sarma, Axial anomaly and longitudinal magnetoresistance of a generic three-dimensional metal, Phys. Rev. B 92, 075205 (2015).

[21] F. Arnold, C. Shekhar, S.-C. Wu, Y. Sun, R. D. dos Reis, N. Kumar, M. Naumann, M. O. Ajeesh, M. Schmidt, A. G. Grushin, J. H. Bardarson, M. Baenitz, D. Sokolov, H. Borrmann, M. Nicklas, C. Felser, E. Hassinger, and B. Yan, Negative magnetoresistance without well-defined chirality in the Weyl semimetal TaP, Nat. Commun. 7, 11615 (2016).

[22] A. V. Andreev and B. Z. Spivak, Longitudinal Negative Magnetoresistance and Magnetotransport Phenomena in Conventional and Topological Conductors, Phys. Rev. Lett. 120, 026601 (2018).

[23] S. Liang, J. Lin, S. Kushwaha, J. Xing, N. Ni, R. J. Cava, and N. P. Ong, Experimental Tests of the Chiral Anomaly Magnetoresistance in the Dirac-Weyl Semimetals $\mathrm{Na}_{3} \mathrm{Bi}$ and GdPtBi, Phys. Rev. X 8, 031002 (2018).

[24] A. Cortijo, Y. Ferreiros, K. Landsteiner, and M. A. H. Vozmediano, Elastic Gauge Fields in Weyl Semimetals, Phys. Rev. Lett. 115, 177202 (2015).

[25] D. I. Pikulin, A. Chen, and M. Franz, Chiral Anomaly from Strain-Induced Gauge Fields in Dirac and Weyl Semimetals, Phys. Rev. X 6, 041021 (2016).

[26] A. G. Grushin, J. W. F. Venderbos, A. Vishwanath, and R. Ilan, Inhomogeneous Weyl and Dirac Semimetals: Transport in Axial Magnetic Fields and Fermi Arc Surface States from PseudoLandau Levels, Phys. Rev. X 6, 041046 (2016).

[27] We assume inversion symmetry such that the energy difference of the Weyl nodes $b_{0}=0$.

[28] J. Shibata, G. Tatara, and H. Kohno, A brief review of fieldand current-driven domain-wall motion, J. Phys. D 44, 384004 (2011).

[29] G. Tatara, H. Kohno, and J. Shibata, Microscopic approach to current-driven domain wall dynamics, Phys. Rep. 468, 213 (2008).

[30] Y. Araki, Magnetic textures and dynamics in magnetic Weyl semimetals, Ann. der Phys. 532, 1900287 (2020).

[31] Y. Ferreiros, F. J. Buijnsters, and M. I. Katsnelson, Dirac electrons and domain walls: A realization in junctions of ferromagnets and topological insulators, Phys. Rev. B 92, 085416 (2015).

[32] Y. Ferreiros and A. Cortijo, Domain wall motion in junctions of thin-film magnets and topological insulators, Phys. Rev. B 89, 024413 (2014).

[33] K. Nomura and N. Nagaosa, Electric charging of magnetic textures on the surface of a topological insulator, Phys. Rev. B 82, 161401(R) (2010).

[34] Y. Tserkovnyak and D. Loss, Thin-Film Magnetization Dynamics on the Surface of a Topological Insulator, Phys. Rev. Lett. 108, 187201 (2012).

[35] J. Linder, Improved domain-wall dynamics and magnonic torques using topological insulators, Phys. Rev. B 90, 041412(R) (2014).

[36] T. D. C. Bevan, A. J. Manninen, J. B. Cook, J. R. Hook, H. E. Hall, T. Vachaspati, and G. E. Volovik, Momentum creation by vortices in superfluid ${ }^{3} \mathrm{He}$ as a 
model of primordial baryogenesis, Nature (London) 386, 689 (1997).

[37] Lattice constant $a=0.5 \mathrm{~nm}$, hard-axis anisotropy $K_{\perp} / a^{3}=$ $10^{2} \mathrm{~J} / \mathrm{m}^{3}$, domain wall width $\lambda=10 \mathrm{~nm}$, Fermi velocity $v_{F}=$ $5 \times 10^{5} \mathrm{~m} / \mathrm{s}$, intervalley scattering rate $\tau=1 \mathrm{~ns}$, Gilbert damping constant $\alpha=0.01$, and half the length of the Weyl node separation $|\boldsymbol{b}|=0.1 \pi / a$.

[38] R. Rajaraman, Solitons and Instantons: An Introduction to Solitons and Instantons in Quantum Field Theory (North-Holland, Amsterdam, 1987).

[39] M. N. Chernodub, A. Cortijo, A. G. Grushin, K. Landsteiner, and M. A. H. Vozmediano, Condensed matter realization of the axial magnetic effect, Phys. Rev. B 89, 081407(R) (2014).

[40] N. L. Schryer and L. R. Walker, The motion of $180^{\circ}$ domain walls in uniform dc magnetic fields, J. Appl. Phys. 45, 5406 (1974).

[41] D. E. Kharzeev, The chiral magnetic effect and anomalyinduced transport, Prog. Part. Nucl. Phys. 75, 133 (2014).

[42] A. M. J. Schakel, Boulevard of Broken Symmetries: Effective Field Theories of Condensed Matter (World Scientific, Singapore, 2008).

[43] T. L. Gilbert, A phenomenological theory of damping in ferromagnetic materials, IEEE Trans. Magn. 40, 3443 (2004).

[44] S. A. Parameswaran, T. Grover, D. A. Abanin, D. A. Pesin, and A. Vishwanath, Probing the Chiral Anomaly with Nonlocal Transport in Three-Dimensional Topological Semimetals, Phys. Rev. X 4, 031035 (2014).

[45] J. Behrends, A. G. Grushin, T. Ojanen, and J. H. Bardarson, Visualizing the chiral anomaly in Dirac and Weyl semimetals with photoemission spectroscopy, Phys. Rev. B 93, 075114 (2016).

[46] K. Fukushima, D. E. Kharzeev, and H. J. Warringa, Chiral magnetic effect, Phys. Rev. D 78, 074033 (2008).

[47] There is an additional axial contribution to the chiral magnetic effect $\boldsymbol{J}=e^{2} /\left(2 \pi^{2} \hbar^{2}\right) \mu \boldsymbol{B}_{5}$ [55]; this does not affect our results as $B_{z}(x, t)$ is odd around the domain wall center and thus averages to zero.
[48] J. D. Jackson, Classical Electrodynamics, 3rd ed. (Wiley, New York, 1999).

[49] The distance from the domain wall to the detector is $R=\mid \boldsymbol{r}-$ $\boldsymbol{r}^{\prime} \mid$, where $\boldsymbol{r}$ is the detector coordinates and $\boldsymbol{r}^{\prime}$ is the domain wall coordinates. We assume the limit $r^{\prime} \ll r$, such that $R \sim r$, and therefore simply refer to the distance to the detector as $r$.

[50] S. Gustavsson, I. Shorubalko, R. Leturcq, T. Ihn, K. Ensslin, and S. Schön, Detecting terahertz current fluctuations in a quantum point contact using a nanowire quantum dot, Phys. Rev. B 78, 035324 (2008).

[51] See Supplemental Material at http://link.aps.org/supplemental/ 10.1103/PhysRevB.102.241401 for a detailed description of the electromagnetic fields induced by the anomaly and magnetization currents, as well as expressions for the radiated power in the near- and far-field limits, which includes Refs. [48,57,58].

[52] R. Aguado and L. P. Kouwenhoven, Double Quantum Dots as Detectors of High-Frequency Quantum Noise in Mesoscopic Conductors, Phys. Rev. Lett. 84, 1986 (2000).

[53] S. Gustavsson, R. Leturcq, B. Simovič, R. Schleser, T. Ihn, P. Studerus, K. Ensslin, D. C. Driscoll, and A. C. Gossard, Counting Statistics of Single Electron Transport in a Quantum Dot, Phys. Rev. Lett. 96, 076605 (2006).

[54] J. Govenius, R. E. Lake, K. Y. Tan, and M. Möttönen, Detection of Zeptojoule Microwave Pulses Using Electrothermal Feedback in Proximity-Induced Josephson Junctions, Phys. Rev. Lett. 117, 030802 (2016).

[55] K. Landsteiner, Notes on anomaly induced transport, Acta Phys. Pol., B 47, 2617 (2016).

[56] J. Behrends, S. Roy, M. H. Kolodrubetz, J. H. Bardarson, and A. G. Grushin, Landau levels, Bardeen polynomials, and Fermi arcs in Weyl semimetals: Lattice-based approach to the chiral anomaly, Phys. Rev. B 99, 140201(R) (2019).

[57] R. Fitzpatrick, Maxwell's Equations and the Principles of Electromagnetism, 1st ed. (Infinity Science Press, Hingham, MA, 2008).

[58] S. Gregson, J. McCormick, and C. Parini, Principles of Planar Near-Field Antenna Measurements, 1st ed. (The Institution of Engineering and Technology, London, 2007). 